\title{
ASSESSING THE RELIABILITY FUNCTION OF NANOCOMPONENTS
}

\author{
NADER EBRAHIMI, ${ }^{*}$ Northern Illinois University
}

\begin{abstract}
A nanocomponent is a collection of atoms arranged to a specific design in order to achieve a desired function with an acceptable performance and reliability. The type of atoms, the manner in which they are arranged within the nanocomponent, and their interrelationship have a direct effect on the nanocomponent's reliability (survival) function. In this paper we propose models based on the notion of a copula that are used to describe the relationship between the atoms of a nanocomponent. Having defined these models, we go on to construct a 'nanocomponent' model in order to obtain the reliability function of a nanocomponent.
\end{abstract}

Keywords: Copula function; Farlie-Gumbel-Morgenstern copula; Gaussian copula; isotropic covariance function; Markov random field; multivariate hazard gradient; reliability function; survival function

2010 Mathematics Subject Classification: Primary 60N05

Secondary 60G55; 90B25

\section{Introduction}

In the fields of nanoscience and nanotechnology much attention has been given to the dual problem of designing nanocomponents with novel physical properties and how they can be fabricated; see Ebrahimi (2008), Ebrahimi (2010), and the references cited therein for more details. Receiving less attention has been the question of the nanocomponent reliability: how does a nanocomponent fail and how long does a nanocomponent survive under typical operation conditions? Today, high reliability is necessary to guarantee the advancement and utilization of nanocomponents due to the fact that they account for a high proportion of costs of newly designed nanosystems and multiscale systems. Recently, Ebrahimi (2008), (2010) developed general methodologies to assess the nanocomponent's limiting reliability from its atoms. In both papers the author was concerned with the nanocomponent reliability at a single instant in time. However, in many practical applications we are interested in the nanocomponent reliability for the interval $[0, t]$, i.e. the probability that the nanocomponent functions successfully throughout the interval $[0, t]$. It is usually referred to as the reliability function or the survival function.

Consider a nanocomponent consisting of $N$ atoms. The nanocomponent can fail because its atoms rearrange. Usually, displacement occurs due to an atom being attracted or repelled by another atom. In order to explore complex patterns of $N$ atoms in three dimensions, assume that there is a set of spatial locations $\left\{s_{i} ; i=1, \ldots, N\right\}$, where $s_{i}=\left(u_{i}, v_{i}, w_{i}\right)$ denotes the location of atom $i$ in space. Here $u_{i}=1, \ldots, N_{1}, v_{i}=1, \ldots, N_{2}, w_{i}=1, \ldots, N_{3}$, and $N=N_{1} N_{2} N_{3}$. To indicate the state of each atom, define the nonnegative continuous random

Received 14 April 2009; revision received 25 October 2010.

* Postal address: Division of Statistics, Northern Illinois University, DeKalb, IL 60115, USA.

Email address: nader@math.niu.edu 
variable $T\left(s_{i}\right)$, where $T\left(s_{i}\right)$ represents the time that the atom at location $s_{i}, i=1, \ldots, N$, is displaced.

Assessing the reliability function of a nanocomponent requires a model for the joint behaviors of the random variables $T\left(s_{1}\right), \ldots, T\left(s_{N}\right)$. For example, suppose that a nanocomponent fails if at least one atom is displaced. Then the reliability function of the nanocomponent is simply given by $R_{N}(t)=\mathrm{P}\left(T\left(s_{i}\right)>t\right.$ for $\left.i=1,2, \ldots, N\right)$. It is clear that specification of the joint survival distribution, $\mathrm{P}\left(T\left(s_{i}\right)>t_{i}, i=1,2, \ldots, N\right)$, which gives such a probability requires full information about the marginal survival functions, $\mathrm{P}\left(T\left(s_{i}\right)>t_{i}\right), i=1,2, \ldots, N$, and dependence between all the atoms. The objective of this paper is to focus on one particular aspect, namely developing models for the joint survival distribution of the random variables $T\left(s_{1}\right), \ldots, T\left(s_{N}\right)$ when partial information about the dependence between atoms is given. Using these models, we then obtain the reliability function for a specific 'nanocomponent' model.

This paper is organized as follows. In Section 2 we present the definitions and notation used in the subsequent sections. In Section 3 we describe models for the joint distribution of $T\left(s_{1}\right), \ldots, T\left(s_{N}\right)$ and provide some insight into these models. For a specific 'nanocomponent' structure, in Section 4 we use the models of Section 3 and provide the reliability function for this structure.

\section{Preliminaries}

To construct a joint survival distribution for $T\left(s_{1}\right), \ldots, T\left(s_{N}\right)$, we concentrate on the notion of a copula, well known for some time within the statistical literature. The standard 'operational' definition of a copula is a multivariate distribution function defined on the unit cube $[0,1]^{p}$, with uniformly distributed marginals. Here $p$ is the number of random variables whose behaviors we wish to understand. The following definition formalizes the notion of a copula.

Definition 1. Let $U_{1}, \ldots, U_{p}$ be $p$ uniform random variables with joint multivariate distribution

$$
C\left(u_{1}, \ldots, u_{p}\right)=\mathrm{P}\left(U_{1} \leq u_{1}, \ldots, U_{p} \leq u_{p}\right) .
$$

The function $C$ is referred to as a copula function which is defined from $[0,1]^{p} \rightarrow[0,1]$.

The function $C\left(u_{1}, \ldots, u_{p}\right)$ defined in (1) is a multivariate distribution whose marginals are all uniform over the interval $(0,1)$. The concept of a copula is relatively simple and, by the celebrated Skylar's theorem (see Skylar (1959)), it serves as an elegant approach for imposing a dependence structure on predetermined marginal distributions. Specifically, let $S$ be a $p$-dimensional joint survival distribution function with marginals $\bar{F}_{1}, \ldots, \bar{F}_{p}$. Then there exists a $p$-dimensional copula such that, for all $\left(x_{1}, \ldots, x_{p}\right)$ in the domain of $S$,

$$
S\left(x_{1}, \ldots, x_{p}\right)=\mathrm{P}\left(X_{1}>x_{1}, \ldots, X_{p}>x_{p}\right)=C\left(\bar{F}_{1}\left(x_{1}\right), \ldots, \bar{F}_{p}\left(x_{p}\right)\right) .
$$

That is, $C\left(\bar{F}_{1}\left(x_{1}\right), \ldots, \bar{F}_{N}\left(x_{p}\right)\right)$ gives a representation of the survival function $S\left(x_{1}, \ldots, x_{p}\right)$. Here $\bar{F}_{i}\left(x_{i}\right)=\mathrm{P}\left(X_{i}>x_{i}\right), i=1, \ldots, p$, and $X_{1}, \ldots, X_{p}$ is a sequence of $p$ random variables. For more details about the notion of a copula and its applications, see Nelson (2006), Yan (2007), Frees and Valdez (2008), and the many references therein.

In our situation, with the copula construction in (1), we can select arbitrary marginal survival distribution functions $\bar{F}_{i}(t)=\mathrm{P}\left(T\left(s_{i}\right)>t_{i}\right), i=1, \ldots, N$. Then, with $p=N$,

$$
C\left(\bar{F}_{1}\left(t_{1}\right), \ldots, \bar{F}_{N}\left(t_{N}\right)\right)=\mathrm{P}\left(T\left(s_{1}\right)>t_{1}, \ldots, T\left(s_{N}\right)>t_{N}\right) .
$$


That is, the copula function $C$ can be used to model the joint survival function of $T\left(s_{1}\right), \ldots$, $T\left(s_{N}\right)$. Note that the function $C$ in (2) is defined from $[0,1]^{N}$ to $[0,1]$.

For imposing a dependence structure on $\bar{F}_{1}, \bar{F}_{2}, \ldots, \bar{F}_{p}$, two frequently used copula families are the elliptical copula family and the Farlie-Gumbel-Morgenstern (FGM) copula family. They are described below.

Definition 2. Let $F$ be the multivariate distribution of an elliptical distribution, let $F_{i}$ be the distribution of the $i$ th margin, and let $F_{i}^{-1}, i=1, \ldots, p$, be its inverse (quantile) function. Then, the elliptical copula is determined by $F$ and its marginals $F_{1}, \ldots, F_{p}$, and is given by

$$
C\left(u_{1}, \ldots, u_{p}\right)=F\left[F_{1}^{-1}\left(u_{1}\right), \ldots, F_{p}^{-1}\left(u_{p}\right)\right] .
$$

In (3) if $F$ is the $p$-variate standard multivariate normal distribution with correlation matrix $\boldsymbol{R}$, then we get the Gaussian copula. That is, the Gaussian copula is given by

$$
C_{\text {Gaussian }}\left(u_{1}, \ldots, u_{p} ; \boldsymbol{R}\right)=\Phi_{\boldsymbol{R}}\left(\Phi^{-1}\left(u_{1}\right), \ldots, \Phi^{-1}\left(u_{p}\right)\right)
$$

where

$$
\begin{gathered}
\Phi_{\boldsymbol{R}}\left(x_{1}, \ldots, x_{p}\right)=\int_{-\infty}^{x_{1}} \cdots \int_{-\infty}^{x_{p}} \frac{1}{(2 \pi)^{p / 2}|\boldsymbol{R}|^{1 / 2}} \exp \left[-\frac{1}{2} z^{\top} \boldsymbol{R}^{-1} z\right] \mathrm{d} z_{1} \cdots d z_{p} \\
\Phi(x)=\int_{-\infty}^{x} \frac{1}{\sqrt{2 \pi}} \exp \left[\frac{-z^{2}}{2}\right] \mathrm{d} z
\end{gathered}
$$

$z^{\top}=\left(z_{1}, \ldots, z_{p}\right)$, and $z^{\top}$ is the transpose of $z$.

Definition 3. The function $C:[0,1]^{p} \rightarrow[0,1]$ defined by

$$
C\left(u_{1}, \ldots, u_{p}\right)=\left(\prod_{i=1}^{p} u_{i}\right)\left[1+\sum_{k=2}^{p} \sum_{1 \leq j_{1}<\cdots<j_{k} \leq p} \theta\left(j_{1}, \ldots, j_{k}\right)\left(1-u_{j_{1}}\right) \cdots\left(1-u_{j_{k}}\right)\right]
$$

is called the $p$-dimensional FGM copula. In (5), the $\theta\left(j_{1}, \ldots, j_{k}\right)$ s are some known parameters.

Throughout this paper, we use the Gaussian copula and a slightly modified version of the FGM copula to impose a dependence structure on $\bar{F}_{1}, \ldots, \bar{F}_{N}$. We cannot overemphasize the fact that these two families are but two of many different families of copula that can be used in practice to model dependence among atoms. We simply use them to illustrate our methodology.

\section{Modeling the joint behavior of $T\left(s_{1}\right), \ldots, T\left(s_{N}\right)$}

Without considering the interactions between atoms, the joint survival distribution of $T\left(s_{1}\right)$, $\ldots, T\left(s_{N}\right)$ is simply $\prod_{i=1}^{N} \mathrm{P}\left(T\left(s_{i}\right)>t_{i}\right)$. Considering interactions among atoms, Markov random field (MRF) theory provides a convenient and consistent way to model interactions via a neighborhood system. Here any random variable, say $T\left(s_{i}\right)$, is assumed to be independent of all other immediate neighbor random variables. For points in a plane, we define a pair to be immediate neighbors if they are closer than a given distance; see Besag (1974) for more details. Let $D(i)=\left\{j: j\right.$ is the neighbor of the atom located at $\left.s_{i}\right\}, i=1, \ldots, N$. To specify the joint distribution of $T\left(s_{1}\right), \ldots, T\left(s_{N}\right)$, we assume that $\left\{T\left(s_{1}\right), \ldots, T\left(s_{N}\right)\right\}$ has the MRF property. Assumption 1 below formalizes the notion of an MRF. 
Assumption 1. (MRF property.) Given any particular atom, say the atom located at $s_{i}$, we assume that

$$
\begin{aligned}
& \mathrm{P}\left(T\left(s_{i}\right)>t_{i} \mid T\left(s_{j}\right)=t_{j}, j=1,2, \ldots, N \text { and } j \neq i\right) \\
& \quad=\mathrm{P}\left(T\left(s_{i}\right)>t_{i} \mid T\left(s_{j}\right)=t_{j}, j \in D(i)\right), \quad i=1, \ldots, N .
\end{aligned}
$$

That is, the conditional survival distribution of $T\left(s_{i}\right)$ depends only on the behaviors of its neighbors. by

Define the distance between two atoms located at $s_{i}=\left(u_{i}, v_{i}, w_{i}\right)$ and $s_{j}=\left(u_{j}, v_{j}, w_{j}\right)$

$$
m\left(s_{i}, s_{j}\right)=\sqrt{\left(u_{i}-u_{j}\right)^{2}+\left(v_{i}-v_{j}\right)^{2}+\left(w_{i}-w_{j}\right)^{2}} .
$$

For simplicity, throughout this paper, we take, for $i=1, \ldots, N$,

$D_{1}(i)=\left\{j: m\left(s_{i}, s_{j}\right)=1\right.$, i.e. the distance between the atom located at $s_{i}$ and its neighbors is 1 \}

and

$$
\mathrm{P}\left(T\left(s_{i}\right)>t_{i} \mid T\left(s_{j}\right)=t_{j}, j=1, \ldots, N, j \neq i\right)=\mathrm{P}\left(T\left(s_{i}\right)>t_{i} \mid T\left(s_{j}\right)=t_{j}, j \epsilon D_{1}(i)\right) .
$$

We consider the following two scenarios.

Scenario 1. In addition to the MRF property, we assume that

$$
K_{1}\left(m\left(s_{i}, s_{j}\right)\right)=\operatorname{cov}\left(T\left(s_{i}\right), T\left(s_{j}\right)\right)=\sigma^{2} \exp \left[-\theta m\left(s_{i}, s_{j}\right)\right], \quad i, j=1, \ldots, N,
$$

where $m\left(s_{i}, s_{j}\right)$ is given by (7) , $\sigma^{2}=\operatorname{var}\left(T\left(s_{i}\right)\right)$, and $\theta \geq 0$. That is, we assume that $\left\{T\left(s_{1}\right), \ldots, T\left(s_{N}\right)\right\}$ is isotropic (see Schabenberger and Gotway $(2005, p .45)$ ), and that the correlation between any two atoms decreases as the distance between them increases. In fact, using (9) and (10), under this scenario, $\operatorname{cov}\left(T\left(s_{i}\right), T\left(s_{j}\right)\right)=0$ if $j$ is not in $D_{1}(i)$. If $j$ is in $D_{1}(i)$ then $\operatorname{cov}\left(T\left(s_{i}\right), T\left(s_{j}\right)\right)=\sigma^{2} \exp [-\theta]$.

Scenario 2. Assume that

$$
\mathrm{P}\left(T\left(s_{i}\right)>t_{i}, T\left(s_{j}\right)>t_{j}\right)=\mathrm{P}\left(T\left(s_{i}\right)>t_{i}\right) \mathrm{P}\left(T\left(s_{j}\right)>t_{j}\right)
$$

for all $i, j=1, \ldots, N$ such that $m\left(s_{i}, s_{j}\right) \geq \gamma$. Here $\gamma$ is a known positive number.

From (11), it is clear that, under Scenario 2, $K_{2}\left(m\left(s_{i}, s_{j}\right)\right)=\operatorname{cov}\left(T\left(s_{i}\right), T\left(s_{j}\right)\right)=0$ if $m\left(s_{i}, s_{j}\right) \geq \gamma$. That is, here $K_{2}$ is a tapered covariance function of compact support. The idea of tapering here is to reduce the covariances to 0 at large distances.

Our first task is to develop a model for the joint survival function of $T\left(s_{1}\right), \ldots, T\left(s_{N}\right)$ under Scenario 1.

Model 1. Assume that

$$
\bar{F}_{i}(t)=\mathrm{P}\left(T\left(s_{i}\right)>t\right)=\bar{F}(t), \quad i=1, \ldots, N .
$$

Note that this assumption is just for convenience and can be easily extended to the nonidentical case. We formulate the model as follows. 
Let $Z\left(s_{1}\right), \ldots, Z\left(s_{N}\right)$ be jointly normal with mean vector zero, variance 1 , and isotropic covariance function $K_{1}(h)=\exp [-\theta h]$; see (10). Also, let $\left\{Z\left(s_{1}\right), \ldots, Z\left(s_{N}\right)\right\}$ be an MRF satisfying (9). Suppose that $C_{\mathrm{Gaussian}}\left(u_{1}, \ldots, u_{N}\right)$ is the corresponding Gaussian copula; see (4). Then our proposed model for the joint survival function is given by

$$
\mathrm{P}\left(T\left(s_{i}\right)>t_{i}, i=1, \ldots, N\right)=C_{\text {Gaussian }}\left(\Phi^{-1}\left(\bar{F}\left(t_{1}\right)\right), \ldots, \Phi^{-1}\left(\bar{F}\left(t_{N}\right)\right)\right) .
$$

Note that in (12), $C$ is defined from $[0,1]^{N} \rightarrow[0,1]$ and it is obtained purely from the dependence structure between atoms.

The following theorem provides some information about the behavior of the sequence $Z\left(s_{1}\right), \ldots, Z\left(s_{N}\right)$.

Theorem 1. Given any particular atom, say the atom located at $s_{i}$,

(a) $\mathrm{E}\left(Z\left(s_{i}\right) \mid Z\left(s_{j}\right), j=1, \ldots, N, j \neq i\right)=b_{i} \sum_{j \in D_{1}(i)} Z\left(s_{j}\right)$,

(b) $\operatorname{var}\left(Z\left(s_{i}\right) \mid Z\left(s_{j}\right), j=1, \ldots, N, j \neq i\right)=d_{i}$,

where

$$
\begin{aligned}
& b_{i}= \begin{cases}\frac{\exp [-\theta]}{1+\left(\left|D_{1}(i)\right|-1\right) \exp [-\theta]} & \text { if }\left|D_{1}(i)\right| \geq 1, \\
0 & \text { if }\left|D_{1}(i)\right|=0,\end{cases} \\
& d_{i}= \begin{cases}1-\frac{\left|D_{1}(i)\right| \exp [-2 \theta]}{1+\left(\left|D_{1}(i)\right|-1\right) \exp [-\theta]} & \text { if }\left|D_{1}(i)\right| \geq 1, \\
1 & \text { if }\left|D_{1}(i)\right|=0,\end{cases}
\end{aligned}
$$

where $\left|D_{1}(i)\right|$ is the number of elements in $D_{1}(i)$, and $D_{1}(i)$ is given by (8).

Proof. The proof follows from Equation (4.6) of Johnson and Wichern (2007) and the fact that $\left\{Z\left(s_{1}\right), \ldots, Z\left(s_{N}\right)\right\}$ is an MRF with covariance structure given by (10) and $\sigma^{2}=1$.

As an application of Theorem 1, suppose that the atom located at $s_{i}$ has three neighbors located at $s_{j}, s_{j^{\prime}}$, and $s_{j^{\prime \prime}}$. Then

$$
\begin{gathered}
\mathrm{E}\left(Z\left(s_{i}\right) \mid Z\left(s_{\ell}\right), \ell=1, \ldots, N, \ell \neq i\right)=\frac{\exp [-\theta]}{1+2 \exp [-\theta]}\left[Z\left(s_{j}\right)+Z\left(s_{j^{\prime}}\right)+Z\left(s_{j^{\prime \prime}}\right)\right] \\
\text { and } \operatorname{var}\left(Z\left(s_{i}\right) \mid Z\left(s_{\ell}\right), \ell=1, \ldots, N, \ell \neq i\right)=1-\frac{3 \exp [-2 \theta]}{1+2 \exp [-\theta]}
\end{gathered}
$$

From (12), we can show that the joint density function of $T\left(s_{1}\right), \ldots, T\left(s_{N}\right)$ under Model 1 is

$$
f\left(t_{1}, \ldots, t_{N}\right)=\left[\prod_{i=1}^{N} f\left(t_{i}\right)\right]\left[c_{\text {Gaussian }}\left(\bar{F}\left(t_{1}\right), \ldots, \bar{F}\left(t_{i}\right), \ldots, \bar{F}\left(t_{N}\right)\right)\right],
$$

where

$$
c_{\text {Gaussian }}\left(u_{1}, \ldots, u_{N}\right)=\frac{1}{|\boldsymbol{R}|^{1 / 2}} \exp \left[-\frac{1}{2} \boldsymbol{w}_{1}^{\top}\left[\boldsymbol{R}^{-1}-\boldsymbol{I}\right] \boldsymbol{w}_{1}\right],
$$

$\boldsymbol{w}_{1}^{\top}=\left(\Phi^{-1}\left(u_{1}\right), \ldots, \Phi^{-1}\left(u_{N}\right)\right), \boldsymbol{I}$ is the $N \times N$ identity matrix, $f(u)=-\mathrm{d} \bar{F}(u) / \mathrm{d} u$, and $\boldsymbol{R}$ is the covariance function, which describes the interactions between atoms and is obtained 
from (10), with $\sigma^{2}=1$ and assuming that the MRF property holds. In (15) $c$ is a function defined from $[0,1]^{N} \rightarrow[0,1]$.

The multivariate hazard gradient $\boldsymbol{h}^{*}\left(t_{1}, \ldots, t_{N}\right)$ of $T\left(s_{1}\right), \ldots, T\left(s_{N}\right)$ under Model 1 , see Johnson and Kotz (1975) for a definition of the multivariate hazard gradient, is given by

$$
\boldsymbol{h}^{*}\left(t_{1}, \ldots, t_{N}\right)=\left(h_{1}^{*}\left(t_{1}, \ldots, t_{N}\right), h_{2}^{*}\left(t_{1}, \ldots, t_{N}\right), \ldots, h_{N}^{*}\left(t_{1}, \ldots, t_{N}\right)\right),
$$

where $\boldsymbol{h}^{*}$ is an $N$-dimensional row vector and

$$
h_{i}^{*}\left(t_{1}, \ldots, t_{N}\right)=-\frac{\partial}{\partial t_{i}}\left[\log \mathrm{P}\left(T\left(s_{i}\right)>t_{i}, i=1, \ldots, N\right)\right] .
$$

Note that $h_{i}^{*}$, which is defined from $[0, \infty]^{N} \rightarrow[0, \infty]$, simply denotes the punctual displacement probability for the atom located at $s_{i}$ at time $t_{i}$ given that other atoms located at $s_{1}, \ldots$, $s_{i-1}, s_{i-1}, \ldots, s_{N}$ are still in their places at times $t_{1}, \ldots, t_{i-1}, t_{i+1}, \ldots, t_{N}$, respectively.

From (15), (16), and Theorem 1, we can easily show that

$$
\begin{aligned}
h_{i}^{*}\left(t_{1}, \ldots, t_{N}\right)= & \frac{f\left(t_{i}\right)(2 \pi)^{1 / 2} \exp \left[\left(\Phi^{-1}\left(\bar{F}\left(t_{i}\right)\right)\right)^{2} / 2\right]}{\Phi_{\boldsymbol{R}}\left(\Phi^{-1}\left(\bar{F}\left(t_{i}\right)\right), i=1, \ldots, N\right)} \\
& \times \int \cdots \int \frac{1}{(2 \pi)^{N / 2}|\boldsymbol{R}|^{1 / 2}} \exp \left[-\frac{1}{2} \boldsymbol{w}^{\top} \boldsymbol{R}^{-1} \boldsymbol{w}\right] \mathrm{d} \boldsymbol{w}^{*},
\end{aligned}
$$

where

$$
\begin{gathered}
\boldsymbol{w}^{* \top}=\left(w\left(s_{1}\right), \ldots, w\left(s_{i-1}\right), w\left(s_{i+1}\right), \ldots, w\left(s_{N}\right)\right), \\
\boldsymbol{w}^{\top}=\left(w\left(s_{1}\right), \ldots, w\left(s_{i-1}\right), \Phi^{-1}\left(\bar{F}\left(t_{i}\right)\right), w\left(s_{i+1}\right), \ldots, w\left(s_{N}\right)\right),
\end{gathered}
$$

and the integral is over $-\infty$ to $\Phi^{-1}\left(\bar{F}\left(t_{j}\right)\right), j=1, \ldots, N, j \neq i$. Consequently, the righthand side becomes

$$
\begin{aligned}
\lambda\left(t_{i}\right) \bar{F}\left(t_{i}\right)(2 \pi)^{1 / 2} \exp \left[\frac{1}{2}\left(\Phi^{-1}\left(\bar{F}\left(t_{i}\right)\right)\right)^{2}\right] & \\
\times \frac{1}{\Phi_{\boldsymbol{R}}\left(\Phi^{-1}\left(\bar{F}\left(t_{i}\right)\right), i=1,2, \ldots, N\right)} \int \cdots \int & \frac{1}{(2 \pi)^{N / 2} d_{i}^{1 / 2}\left|\boldsymbol{R}^{*}\left(s_{i}\right)\right|^{1 / 2}} \\
& \times \exp \left[-\frac{\left(\Phi^{-1}\left(\bar{F}\left(t_{i}\right)\right)-b_{i}\right)^{2}}{2 d_{i}}\right] \\
& \times \exp \left[-\frac{\boldsymbol{w}^{* \top}\left(\boldsymbol{R}^{*}\left(s_{i}\right)\right)^{-1} \boldsymbol{w}^{*}}{2}\right] \mathrm{d} \boldsymbol{w}^{*},
\end{aligned}
$$

where $\boldsymbol{R}^{*}\left(s_{i}\right)$ is the correlation matrix between atoms located at $s_{j}$ and $s_{j^{\prime}}, j, j^{\prime} \neq i, j=$ $1, \ldots, N, j^{\prime}=1, \ldots, N$, and $b_{i}$ and $d_{i}$ are given in (13) and (14), respectively.

In general, it is possible to compute the joint survival function and multivariate gradient hazard function provided that (12) and (16) can be evaluated. However, in many cases it is very difficult to calculate these equations. In such cases we can seek upper and lower bounds for (12) as well as an approximation for $\boldsymbol{h}^{*}$ in (16).

The following theorem gives the upper and lower bounds for the joint survival function of $T\left(s_{1}\right), \ldots, T\left(s_{N}\right)$. 
Theorem 2. Under Model 1,

$$
\prod_{i=1}^{N} \bar{F}\left(t_{i}\right) \leq \mathrm{P}\left(T\left(s_{i}\right)>t_{i}, i=1, \ldots, N\right) \leq \mathrm{P}\left(T^{*}\left(s_{i}\right)>t_{i}, i=1, \ldots, N\right),
$$

where the joint survival function of $T^{*}\left(s_{1}\right), \ldots, T^{*}\left(s_{N}\right)$ is obtained by assuming that $K(h)=$ $\exp [-\theta]$ instead of $K_{1}(h)$ in (10). That is,

$$
\begin{aligned}
\mathrm{P}\left(T^{*}\left(s_{i}\right)>t_{i}, i\right. & =1, \ldots, N) \\
=\frac{1}{\sqrt{2 \pi}} \int_{-\infty}^{\infty} \exp \left[-\frac{x^{2}}{2}\right] & \\
& \times \prod_{i=1}^{N}\left[\Phi(1-\exp [-\theta])^{-1 / 2} \Phi^{-1}\left(\bar{F}\left(t_{i}\right)\right)+\left(\frac{\exp [-\theta]}{1-\exp [-\theta]}\right)^{1 / 2} x\right] \mathrm{d} x .
\end{aligned}
$$

Proof. Suppose that $W_{1}, \ldots, W_{k}$ are random variables having a $k$-variate multivariate normal distribution with mean vector $\mathbf{0}$, variance 1 , and, under the probability law $\mathrm{P}_{\boldsymbol{L}_{1}}$, the correlation matrix $\boldsymbol{L}_{1}=\left\{\ell_{i r}(1)\right\}$, and, under the probability law $\mathrm{P}_{\boldsymbol{L}_{2}}$, the correlation matrix $\boldsymbol{L}_{2}=\left\{\ell_{i r}(2)\right\}$. If the correlation $\ell_{i r}(1) \leq \ell_{i r}(2)$ for all $i$ and $r$, then it is known that

$$
\mathrm{P}_{L_{1}}\left(W_{1} \leq c_{1}, \ldots, W_{k} \leq c_{k}\right) \leq \mathrm{P}_{\boldsymbol{L}_{2}}\left(W_{1} \leq c_{1}, \ldots, W_{k} \leq c_{k}\right)
$$

for any numbers $c_{1}, c_{2}, \ldots, c_{k}$; see Slepian (1962) for more details. The result now follows from Tong (1990, p. 193).

The following example shows an interesting application of Theorem 2.

Example 1. Consider a nanocomponent with three atoms, i.e. $N=3$, such that $\theta=\frac{1}{2}$ and $\bar{F}(t)=\exp [-t]$. Suppose that the lifetime of the nanocomponent $T=\min \left(T\left(s_{1}\right), T\left(s_{2}\right)\right.$, $T\left(s_{3}\right)$ ) and that the joint survival function of $T\left(s_{1}\right), T\left(s_{2}\right)$, and $T\left(s_{3}\right)$ can be modeled by (12) (Model 1). Then, the reliability function is $R_{3}(t)=\mathrm{P}(T>t)=\mathrm{P}\left(T\left(s_{1}\right)>t\right.$, $\left.T\left(s_{2}\right)>t, T\left(s_{3}\right)>t\right)$ and, from Theorem 2 ,

$$
\begin{aligned}
R_{3}(t) \leq \frac{1}{\sqrt{2 \pi}} \int_{-\infty}^{\infty} \exp \left[-\frac{x^{2}}{2}\right][ & \Phi\left(1-\exp \left[-\frac{1}{2}\right]\right)^{-1 / 2} \Phi^{-1}(\exp [-t]) \\
& \left.+\left(\frac{\exp [-1 / 2]}{1-\exp [-1 / 2]}\right)^{1 / 2} x\right]^{3} \mathrm{~d} x
\end{aligned}
$$

In Table 1 we present results for the upper bound of the reliability function $R_{3}(t)$ for various $t$.

Suppose that the lifetime $T$ is measured in years. In view of Table 1 , the chance that the nanocomponent survives for more than, say, 0.5 years ( 6 months), is less than 0.33 .

Under Model 1, we can also approximate the joint survival function of $T\left(s_{1}\right), \ldots, T\left(s_{N}\right)$ and the multivariate hazard gradient $\boldsymbol{h}^{*}\left(t_{1}, \ldots, t_{N}\right)$ using the following procedure.

The multivariate Gaussian copula in (4) can be approximated by a Taylor expansion:

$$
\begin{aligned}
C_{\text {Gaussian }}\left(u\left(s_{1}\right), \ldots, u\left(s_{N}\right)\right)=\prod_{i=1}^{N} u\left(s_{i}\right)+\sum_{i=1}^{N} \sum_{j=1}^{N} & K_{1}\left(m\left(s_{i}, s_{j}\right)\right) \\
& \times \phi\left(\Phi^{-1}\left(u\left(s_{i}\right)\right)\right) \phi\left(\Phi^{-1}\left(u\left(s_{j}\right)\right)\right) .
\end{aligned}
$$


TABLE 1: Upper bounds for $R_{3}(t)$.

\begin{tabular}{cc}
\hline$t$ & $R_{3}(t)$ \\
\hline 0.1 & 0.78 \\
0.2 & 0.62 \\
0.3 & 0.50 \\
0.4 & 0.40 \\
0.5 & 0.33 \\
0.6 & 0.27 \\
0.7 & 0.22 \\
0.8 & 0.18 \\
0.9 & 0.15 \\
1.0 & 0.12 \\
\hline
\end{tabular}

In (17), $K_{1}(h)$ is defined by (10), with $\sigma^{2}=1$ and $\phi(x)=\sqrt{(1 / 2 \pi)} \exp \left[-x^{2} / 2\right]$. Thus, taking $V=\sum_{i=1}^{N} \sum_{j=1}^{N} \exp \left[-\theta m\left(s_{i}, s_{j}\right)\right]$, applying (17), and using the fact that $\mathrm{d} \phi\left(\Phi^{-1}(\bar{F}(t))\right) / \mathrm{d} t$ $=f(t) \Phi^{-1}(\bar{F}(t))$, we obtain

$$
\begin{aligned}
\mathrm{P}\left(T\left(s_{i}\right)>t_{i}, i=1, \ldots, N\right)=\prod_{i=1}^{N} \bar{F}\left(t_{i}\right)+\sum_{i=1}^{N} \sum_{j=1}^{N} & \exp \left[-\theta m\left(s_{i}, s_{j}\right)\right] \\
& \times \phi\left(\Phi^{-1}\left(\bar{F}\left(t_{i}\right)\right)\right) \phi\left(\Phi^{-1}\left(\bar{F}\left(t_{j}\right)\right)\right) .
\end{aligned}
$$

Using (18), we can easily show that

$$
\begin{aligned}
h_{i}^{*}\left(t_{1}, \ldots, t_{N}\right)= & f\left(t_{i}\right)\left[\mathrm{P}\left(T\left(s_{i}\right)>t_{i}, i=1, \ldots, N\right)\right]^{-1} \\
& \times\left[\prod_{j=1, j \neq i}^{N} \bar{F}\left(t_{j}\right)+\sum_{j=1, j \neq i}^{N} K_{1}\left(m\left(s_{i}, s_{j}\right)\right)\left(\Phi^{-1}\left(\bar{F}\left(t_{i}\right)\right)\right) \phi\left(\Phi^{-1}\left(\bar{F}\left(t_{j}\right)\right)\right)\right] .
\end{aligned}
$$

Thus, for $t_{i}=t, i=1, \ldots, N$, and large $N$ (i.e. $N \rightarrow \infty$ ), (19) reduces to

$$
\begin{aligned}
h_{i}^{*}(t, \ldots, t)= & {\left[(\bar{F}(t))^{N}+\left(\phi\left(\Phi^{-1}(\bar{F}(t))\right)^{2}\right) V\right]^{-1} } \\
& \times\left[f(t)(\bar{F}(t))^{N-1}+f(t) \phi\left(\Phi^{-1}(\bar{F}(t))\right) \Phi^{-1}(\bar{F}(t)) \sum_{j=1, j \neq i}^{N} K_{1}\left(m\left(s_{i}, s_{j}\right)\right)\right] \\
= & \frac{f(t)}{\bar{F}(t)} \frac{1+a_{N}}{1+b_{N}} \\
= & \frac{f(t)}{\bar{F}(t)}
\end{aligned}
$$

where $b_{N} \rightarrow \infty$ and $a_{N} / b_{N} \rightarrow 0$ as $N \rightarrow \infty$. That is, the gradient hazard function for any atom is approximately the same as its marginal hazard function.

We now develop a model for the joint survival function of $T\left(s_{1}\right), \ldots, T\left(s_{N}\right)$ by incorporating the assumptions described in Scenario 2. 
Model 2. Assume that $\mathrm{P}\left(T\left(s_{i}\right)>t\right)=\bar{F}(t), i=1, \ldots, N$. The model can be formulated by slightly modifying the FGM copula described in Definition 3. Our proposed modified FGM (MFGM) copula is

$$
C_{\mathrm{MFGM}}\left(u\left(s_{1}\right), \ldots, u\left(s_{n}\right)\right)=\prod_{i=1}^{N} u\left(s_{i}\right)\left[1+\sum_{\ell=1}^{\gamma} \sum_{Q(\ell)} \theta_{\ell}\left(1-u\left(s_{i}\right)\right)\left(1-u\left(s_{j}\right)\right)\right],
$$

where $Q(\ell)=\left\{i\right.$ and $\left.j: m\left(s_{i}, s_{j}\right)=\ell\right\}$. Using the MFGM copula, our proposed model for the joint survival function of $\left.T\left(s_{1}\right), \ldots, T\left(s_{N}\right)\right)$ is given by

$$
\mathrm{P}\left(T\left(s_{1}\right)>t_{1}, \ldots, T\left(s_{N}\right)>t_{N}\right)=C_{\mathrm{MFGM}}\left(\bar{F}\left(t_{1}\right), \ldots, \bar{F}\left(t_{N}\right)\right) .
$$

Again, for simplicity, we assume that $\ell=1$. Thus, (20) reduces to

$$
\mathrm{P}\left(T\left(s_{1}\right)>t_{1}, \ldots, T\left(s_{N}\right)>t_{N}\right)=\prod_{i=1}^{N} \bar{F}\left(t_{i}\right)\left[1+\theta \sum_{Q(1)} F\left(t_{i}\right) F\left(t_{j}\right)\right],
$$

where $F=1-\bar{F}$.

From (21), we can easily show that the joint density function of $T\left(s_{1}\right), \ldots, T\left(s_{N}\right)$ is given by

$$
f\left(t_{1}, \ldots, t_{N}\right)=\prod_{i=1}^{N} f\left(t_{i}\right)\left[1+\theta \sum_{Q(1)}\left(1-2 \bar{F}\left(t_{i}\right)\right)\left(1-2 \bar{F}\left(t_{j}\right)\right)\right] .
$$

Also, from (21), the multivariate hazard gradient for the $T\left(s_{1}\right), \ldots, T\left(s_{N}\right)$ is given by

$$
\boldsymbol{h}^{*}\left(t_{1}, \ldots, t_{N}\right)=\left(h_{1}^{*}\left(t_{1}, \ldots, t_{N}\right), \ldots, h_{N}^{*}\left(t_{1}, \ldots, t_{N}\right)\right),
$$

where

$$
h_{i}^{*}\left(t_{1}, \ldots, t_{N}\right)=\lambda\left(t_{i}\right)\left[1+\frac{\theta \bar{F}\left(t_{i}\right) \sum_{Q_{i}(1)} F\left(t_{j}\right)}{1+\theta \sum_{Q(1)} F\left(t_{i}\right) F\left(t_{j}\right)}\right],
$$

$Q_{i}(1)=\left\{j: m\left(s_{i}, s_{j}\right)=1\right\}$, and $\lambda(u)=f(u) / \bar{F}(u)$ is the marginal hazard function. Suppose that $t_{i}=t$ for $i=1, \ldots, N$. Then (22) reduces to

$$
h_{i}^{*}(t, \ldots, t)=\lambda(t)\left[1+\frac{\theta \bar{F}(t) F(t)\left|Q_{i}(1)\right|}{1+\theta F^{2}(t)|Q(1)|}\right],
$$

where $|A|$ is the number of elements in the set $A$. It should be noted that $h_{i}^{*}(t, \ldots, t)$ in (23) simply denotes the punctual displacement probability for the atom located at $s_{i}$ at time $t$ given that other atoms are still in their places at time $t$.

From (23), it is clear that at $t=0$ and $t=\infty, h_{i}^{*}(t, \ldots, t)=\lambda(0)$ and $\lambda(\infty)$, respectively. Also, for large $N$ (i.e. $N \rightarrow \infty$ ), since $|Q(1)| \rightarrow \infty$ and $\left|Q_{i}(1)\right|$ is finite,

$$
h_{i}^{*}(t, \ldots, t)=\lambda(t)
$$

That is, in Model 2, for large $N$ (i.e. $N \rightarrow \infty$ ), the gradient hazard function for any atom is the same as its marginal hazard function. 


\section{Reliability function of nanocomponents}

In this section we illustrate how Model 1 and Model 2 can be used to obtain the reliability function for a specific 'nanocomponent' model.

Generally speaking, let $T$ be the lifetime of a nanocomponent. Then its reliability function is given by

$$
R_{N}(t)=\mathrm{P}(T>t) .
$$

Suppose that the lifetime of a nanocomponent can be represented by $T=\Psi\left(T\left(s_{1}\right), \ldots, T\left(s_{N}\right)\right)$, where $\Psi$ is some known function defined from $[0, \infty]^{N} \rightarrow[0, \infty]$. We refer to $\Psi$ as the 'nanocomponent' model. In this section we assume that the 'nanocomponent' model $\Psi\left(u_{1}, \ldots, u_{N}\right)=\min _{1 \leq i \leq N} u_{i}$. That is, the nanocomponent will fail if at least one of the atoms is displaced.

Now, it is clear that, under this 'nanocomponent' model,

$$
R_{N}(t)=\mathrm{P}\left(T\left(s_{i}\right)>t, i=1, \ldots, N\right) .
$$

Our goal in this section is to obtain $R_{N}(t)$ under Model 1 and Model 2. We start with Model 2. From (21), (24) reduces to

$$
R_{N}(t)=(\bar{F}(t))^{N}\left(1+\theta F^{2}(t)|Q(1)|\right) .
$$

Theorem 3 below gives a simple approximation to $R_{N}(t)$ in (25) for large $N$ (i.e. $N \rightarrow \infty$ ).

Theorem 3. Assume that there are sequences $a_{N}$ and $b_{N}>0$ of real numbers such that, for all $y$, as $N \rightarrow \infty$,

$$
\lim _{N \rightarrow \infty} N F\left(a_{N}+b_{N} y\right)=w(y)
$$

exists. Then, under Model 2, as $N \rightarrow \infty$,

$$
\lim _{N \rightarrow \infty} R_{N}\left(a_{N}+b_{N} t\right)=\exp [-w(t)]
$$

Proof. Take $z=F\left(a_{N}+b_{N} y\right)$. Using (25), we obtain

$$
R_{N}\left(a_{N}+b_{N} y\right)=(1-z)^{N}\left(1+\theta z^{2}|Q(1)|\right) \leq \exp [-N z]\left(1+\theta z^{2}|Q(1)|\right) .
$$

Let us first consider the case when $w(y)=+\infty$. Then

$$
R_{N}\left(a_{N}+b_{N} y\right) \leq \exp [-w(y)](1+O(w(y))=0
$$

immediately yields $(26)$ (recall that $\exp [-\infty]=0$ ). Note that, for sequences of real positive numbers $c_{N}$ and $d_{N}, d_{N}=O\left(c_{N}\right)$ if there is a positive constant number $M$ such that $d_{N}<M c_{N}$ for all sufficiently large $N$.

Now, consider the case when $w(y)<\infty$. Using Lemma 1.3.1 of Galambos (1978),

$$
\begin{aligned}
\left(\exp [-N z]-(1-z)^{N}\left(\exp \left[2 N z^{2}\right]-1\right)\right)\left(1+\theta z^{2}|Q(1)|\right) & <R_{N}\left(a_{N}+b_{N} y\right) \\
& <\exp [-N z]\left(1+\theta z^{2}|Q(1)|\right)
\end{aligned}
$$

Since $\lim _{N \rightarrow \infty} N z^{2}=1 / N(N z)^{2}=0$, we obtain $\lim _{N \rightarrow \infty}\left(1+\theta z^{2}|Q(1)|\right)=1$ and, therefore,

$$
\lim _{N \rightarrow \infty} R_{N}\left(a_{N}+b_{N} y\right)=\exp [-w(y)] .
$$

This completes the proof. 
The next example illustrates an application of Theorem 3.

Example 2. In Theorem 3, let

$$
\bar{F}(x)=\exp [-\lambda x] .
$$

Then, $F\left(a_{N}+b_{N} x\right)=1-\exp \left[-\lambda\left(a_{N}+b_{N} x\right)\right]$. In order to satisfy the assumption of Theorem 3, take $a_{N}=0$ and $b_{N}=1 / N$; then $\lim N(1-\exp [-\lambda y / N])=\lambda y$. That is, $R_{N}(y / N)=\exp [-\lambda y]$.

Now we turn our attention to Model 1. Using (12), we can easily show that, under this model, (24) reduces to

$$
R_{N}(t)=\Phi_{\boldsymbol{R}}\left(\Phi^{-1}(\bar{F}(t)), \ldots, \Phi^{-1}(\bar{F}(t))\right) .
$$

We now prove the following result.

Theorem 4. Under Model 1, let $b_{N}=(2 \log N)^{-1 / 2}$ and $a_{N}=1 / b_{N}-b_{N}(\log \log N+$ $\log 4 \pi) / 2$. Then, as $N \rightarrow \infty$, the simple approximation to $R_{N}(t)$ in (27) is

$$
R_{N}\left(\bar{F}^{-1}\left(\Phi\left(a_{N}+b_{N} u\right)\right)\right) \rightarrow \exp \left[-\mathrm{e}^{-u}\right], \quad-\infty<u<\infty .
$$

Proof. Using (27) and Theorem 3.8.2 of Galambos (1978), as $N \rightarrow \infty$, we obtain

$$
\begin{aligned}
R_{N}\left(\bar{F}^{-1}\left(\Phi\left(a_{N}+b_{N} u\right)\right)\right) & =\Phi_{\boldsymbol{R}}\left(a_{N}+b_{N} u, \ldots, a_{N}+b_{N} u\right) \\
& =\mathrm{P}\left(\max \left(Z\left(s_{1}\right), \ldots, Z\left(s_{N}\right)\right)<a_{N}+b_{N} u\right) \\
& =\exp \left[-\mathrm{e}^{-u}\right] .
\end{aligned}
$$

This completes the proof.

The following example gives an application of Theorem 4 .

Example 3. Let $\bar{F}(t)=\exp [-\lambda t]$. From Theorem 4 we have

$$
R_{N}\left(-\bar{\lambda}^{1} \log \Phi\left(a_{N}+b_{N} u\right)\right)=\exp \left[-\mathrm{e}^{-u}\right], \quad-\infty<u<\infty .
$$

In (28), suppose that $N=100$ and $\lambda=0.01$. Then, $b_{N}=0.33, a_{N}=2.33$, and $R_{100}(-100 \log \Phi(2.33+0.33 u))=\exp \left[-\mathrm{e}^{-u}\right]$. For example, $R_{100}(0)=1, R_{100}(0.05)=$ $0.95, R_{100}(0.1)=0.87, R_{100}(0.4)=0.7, R_{100}(0.9)=0.37$, and $R_{100}(2.3)=0.066$.

\section{Acknowledgement}

This research was partially supported by the National Security Agency under grant number H98230-11-1-0138.

\section{References}

Besag, J. (1974). Spatial interaction and statistical analysis of lattice systems. J. R. Statist. Soc. B 36, $192-236$.

Ebrahimi, N. (2008). Assessing a linear nanosystem's limiting reliability from its components. J. Appl. Prob. 45, 879-887.

EBrahimi, N. (2010). Assessing 2-dimensional nanocomponent's reliability. IEEE Trans. Reliab. 59, $139-144$.

Frees, E. W. And Valdez, E. A. (2008). Hierarchical insurance claims modeling. J. Amer. Statist. Assoc. 103, 14571469.

Galambos, J. (1978). The Asymptotic Theory of Extreme Order Statistics. John Wiley, New York.

Johnson, N. L. And Kotz, S. (1975). A vector multivariate hazard rate. J. Multivariate Anal. 5, 53-66. 
Johnson, R. A. And Wichern, D. W. (2007). Applied Multivariate Statistical Analysis, 6th edn. Pearson Prentice Hall, Upper Saddle River, NJ.

Nelson, R. B. (2006). An Introduction to Copulas, 2nd edn. Springer, New York.

Schabenberger, O. and Gotway, C. A. (2005). Statistical Methods for Spatial Data Analysis. Chapman and Hall/CRC, Boca Raton, FL.

Sklar, A. (1959). Function de répartition à $n$ dimensions et leurs marges. Publ. Inst. Statist. Univ. Paris 8, $229-231$. SlePIAN, D. (1962). The one-sided barrier problem for Gaussian noise. Bell System Tech. J. 41, 463-501.

Tong, Y. L. (1990). The Multivariate Normal Distribution. Springer, New York.

YAN, J. (2007). Enjoying the joy of copulas: with a package copula. J. Statist. Software 21, 1-21. 